\title{
A comment on the flow and heat transfer past a permeable stretching/shrinking surface in a porous medium: Brinkman model
}

\begin{abstract}
An analytical solution is presented for the boundary-layer flow and heat transfer over a permeable stretching/shrinking surface embedded in a porous medium using the Brinkman model. The problem is seen to be characterized by the Prandtl number Pr, a mass flux parameter $\mathrm{s}$, with $\mathrm{s}>0$ for suction, $\mathrm{s}=0$ for an impermeable surface, and $\mathrm{s}<0$ for blowing, a viscosity ratio parameter $\mathrm{M}$, the porous medium parameter $\Lambda$ and a wall velocity parameter $\lambda$. The analytical solution identifies critical values which agree with those previously determined numerically (Bachok et al. Proceedings of the fifth International Conference on Applications of Porous Media, 2013) and shows that these critical values, and the consequent dual solutions, can arise only when there is suction through the wall, $\mathrm{s}>0$.
\end{abstract}

Keyword: Boundary layer; Heat transfer; Suction/blowing; Stretching/shrinking sheet;

Porous medium; Dual solutions 